\title{
Experimental posterior uveitis. II. Electroretinographic studies
}

\author{
M R STANFORD' AND J ROBBINS ${ }^{2}$ \\ From the Departments of 'Immunology and ${ }^{2}$ Vision Research, the Rayne Institute, UMDS, St Thomas's \\ Campus, Lambeth Palace Road, London SE1 7EH
}

SUMMARY Experimental posterior uveitis was induced by the inoculation of retinal S-antigen into black hooded Lister rats. The time course of the disease was monitored by electroretinography (ERG), and the ERG changes were correlated with clinical signs and underlying pathological damage. The ERG became supernormal in the third week after inoculation, with some loss of temporal resolution (lowered ERG flicker fusion frequency), though the disease was not clinically manifest at this time and histological examination was normal. The ERG became subnormal after 21 days as clinical signs of disease began to appear. This subnormality was associated with focal photoreceptor necrosis, the degree of destruction being proportional to the reduction in the ERG. The ERG recovered with resolution of disease in most cases, though return to control values was uncommon. We suggest that the initial supernormal ERG reflects an underlying biochemical change mediated by the action of anti retinal S-antigen antibodies.

The electroretinogram (ERG) is widely accepted as a useful clinical and experimental technique for the investigation of retinal disease. The ERG is the synchronous and sequential response of the retina to a light stimulus, ${ }^{1}$ and analysis of the component waves allows anatomical localisation of disease processes. ${ }^{23}$ The ERG has been used in the study of patients with posterior uveitis, ${ }^{49}$ and both supernormal and subnormal responses of components of the ERG (usually the b-wave) have been detected. However, although these abnormalities correlated with the extent of disease observed clinically, comparative analysis with the underlying histopathology has been impossible owing to the lack of suitable material. Experimental models of disease are thus required.

Early ERG studies of experimental allergic uveitis (EAU) involved the intravitreal injection of heterologous antigen, ${ }^{10-12}$ but recently the disease has been induced by the injection of antigen at a site remote from the eye, thus avoiding this manipulation. ${ }^{13-15}$ The earliest electrophysiological sign of disease in rodents, which have rod dominant retinae, was an increase in the size of the scotopic b-wave, followed by a reduction and then extinction of the ERG as the disease progressed. In cone rich retinae, such as that Correspondence to Mr M R Stanford, FRCS. found in the pigeon, no change was found in the bwave, but the a-wave and oscillatory potentials became progressively reduced during active disease. ${ }^{10}$ The subnormality of the ERG corresponded to histologically documented destruction of the retinae in all these experimental models.

We investigated the electrophysiological responses of the black hooded Lister rat with inflammatory eye disease induced by a footpad injection of retinal Santigen.$^{16}$ Disease in this animal model is confined to the posterior segment of the eye and shows striking clinical similarities to posterior uveitis in man, especially idiopathic retinal vasculitis. ${ }^{17}$ There is little concomitant vitreous inflammation, and artefacts induced by clouding of the media are largely avoided. The aims of this study were to correlate ERG changes during disease with the underlying pathology and to try to delineate early electrophysiological markers that might predict disease onset.

\section{Materials and methods}

GENERAL EXPERIMENTAL PROCEDURE

A total of 39 male black hooded Lister rats (Bantin and Kingman), weighing 250-300 g were used. Twenty-eight of these were experimental animals and 11 served as controls. At the age of 10 weeks 
experimental animals received $50 \mu \mathrm{g}$ of retinal Santigen emulsified in Freund's complete adjuvant (FCA) enriched with Myobacterium tuberculosis (3 $\mathrm{mg} \mathrm{ml}^{-1}$ ) divided into $0.1 \mathrm{ml}$ aliquots given into each hind footpad. Control animals received $0.1 \mathrm{ml}$ of phosphate buffered saline (PBS) and FCA. The details of the procedure to purify the retinal $S$ antigen by means of size and hydrophobic absorption chromatography have been described elsewhere. ${ }^{18}$ The material used was $80 \%$ pure as assessed by sodium dodecyl sulphate gel electrophoresis (SDSPAGE) and scanning densitometry. All rats were kept on the same shelf under 12-hour light $(60 \mathrm{~cd}$ $\left.\mathrm{m}^{-2}\right)$ /dark cycles. They were fed with water and $R$ and $M$ No. 1 maintenance cubes (SDS).

The animals were divided into three groups. In each group experimental and control animals were weight matched. ERGs were performed at the same time of day for each animal.

Group I. Three experimental and three control animals were used. Each animal had an ERG recorded every two to four days (11 separate recordings/animal) until day 37 after inoculation, when the experiment was terminated.

Group II. Nine experimental animals and four controls were used. ERGs were recorded daily from day 13 to day 20 after inoculation. Experimental animals were killed when their ERG became significantly enhanced as compared with controls. Control animals were killed on day 20 after inoculation.

Group III. A: Five experimental animals had ERG recordings 120 days after inoculation and were then killed. B: Eleven experimental animals and four controls had ERG recordings every 20 days until day 86 after inoculation, when they were killed.

\section{PROCEDURE FOR ELECTRORETINOGRAPHIC INVESTIGATION}

Before each ERG recording all the animals were dark adapted for 30 minutes and anaesthetised with an intraperitoneal injection of ketamine hydrochloride (Ketalar, Parke Davis) $120-180 \mathrm{mg} \mathrm{kg}^{-1}$, which provided a suitable level of anaesthesia for recordings to be made. ${ }^{19}$ The animals were placed in a home built stereotaxic rat holder.

ERGs were recorded with silver electrodes embedded in a Perspex contact lens with an indifferent needle electrode placed in the skin of the cheek. The pupils were dilated with $1 \%$ tropicamide hydrochloride (Smith and Nephew). The responses from each eye were amplified (Electromed AC 3543, band width $0 \cdot 2-100 \mathrm{~Hz}$ ) and displayed on a twin channel curvilinear pen recorder (Electromed MX212). Oscillatory potentials were recorded by passing the signal through a $100 \mathrm{~Hz}$ high pass passive filter, and increasing the top cut to $1000 \mathrm{~Hz}$.
A xenon flash bulb (Nore Microwave NV 615) projected into a dome producing ganzfeld stimulation (maximum intensity at $0.0 \mathrm{log}$ units $=2800 \mathrm{~cd}$ $\mathrm{m}^{-2}$ ) was used to evoke ERGs. Intensity and wavelength of the flash were altered by interposing neutral density and colour (Kodak) filters. The frequency of the flash rate was varied by a function generator (Feedback TWG 500). ERGs were recorded with flashes of different intensities, beginning with the lowest (that is, with $\overline{4} \cdot 0 \log$ units neutral density filter). For each intensity level the stimulation rate was altered from $5 \mathrm{~Hz}$ to $30 \mathrm{~Hz}$ in $5 \mathrm{~Hz}$ steps. Between each change of intensity level a two-minute interval was allowed for recovery from light adaptation. After the above procedure ERGs were also recorded with red (Kodak 29) and blue (Kodak 47B) flashes. In later experiments flicker ERGs were obtained with the highest intensity flashes.

\section{OTHER INVESTIGATIONS}

After the ERG recordings each animal was assessed by slit-lamp biomicroscopy and photographs of the fundus were taken with a fundus camera (Zeiss). Fundus photography also allowed an assessment of the clarity of the ocular media by an independent observer. The recovery of all animals was monitored and all animals were weighed regularly to assess their state of health. At the end of each experimental series, the animal was anaesthetised with urethane $\left(1.5 \mathrm{~g} \mathrm{~kg}^{-1}\right)$ and fluorescein angiography was carried out. The animals were bled by cardiac puncture, killed by cervical dislocation, and the eyes enucleated.

\section{HISTOLOGICAL PROCEDURE}

The enucleated eyes were placed in $4 \%$ glutaraldehyde for 12 hours and then in $10 \%$ neutral buffered formalin for more than 24 hours. The eyes were dehydrated through graded alcohols, cleared in toluene, and embedded in wax. Histological sections were stained with haematoxylin and eosin throughout.

\section{Results}

GROUP I: ERG CHANGES FROM DAY 3 TO 37 AFTER INOCULATION

The main features of the ERG changes observed in experimental animals in the initial study are shown in Fig. 1. In two animals (E1 and E3) the ERG became supernormal prior to the onset of disease, and this change was associated with the appearance of vitreous cells. Subsequently in these two animals the ERG values fell, becorming subnormal in E1 with the onset of clinical disease (disc swelling, periphlebitis, and retinal infiltrates), approaching subnormality in 
control
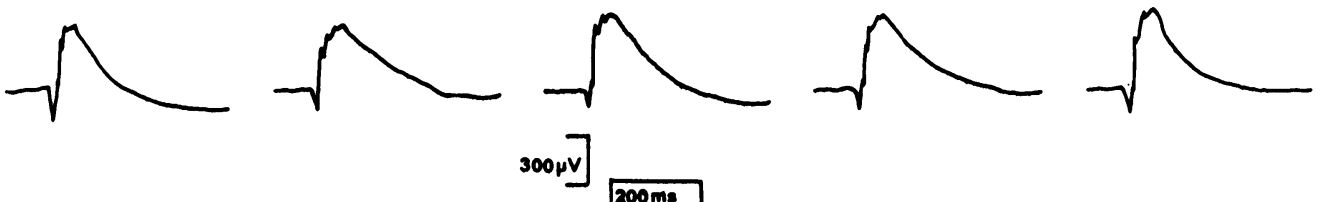

E1<smiles>CCCCC</smiles>
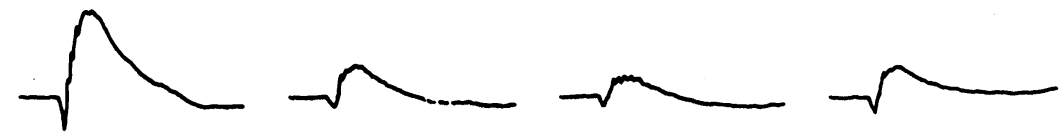

vitreous cells

clinical disease

E2
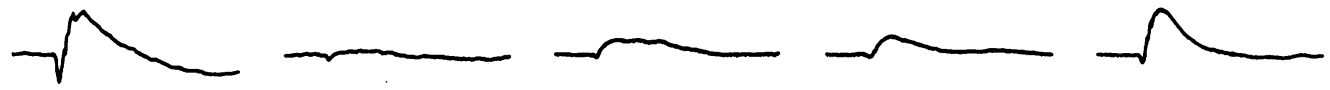

\section{clinical disease}

E3
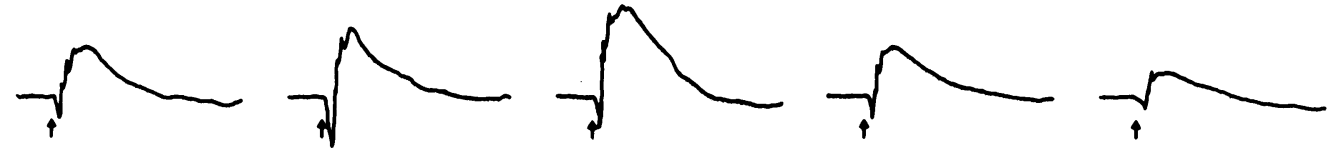

vitreous cells

6

18

26

34

37

\section{Post Inoculation Day (PID)}

Fig. 1 ERGs at the highest light intensity (O log units ( Lu )) for one control animal and three experimental animals (E1, E2, E3) at postinoculation days 6, 18, 26, 34, and 37. Showing the relationship between clinical disease and ERG waveform. Arrows indicate stimulus.

E3. In animal E2 a bilateral posterior uveitis was evident on day 16 , associated with a severely reduced ERG. However, in this animal the ERG recovered as disease settled, though it was still subnormal at the termination of the experiment.

A number of associated features were observed
Fig. 2 The ratio of the $a$ - and $b$ waves (mean $\pm S D$ ) for control (solid line) and experimental (dashed line) animals at days after inoculation. with the above ERG changes. Firstly, there was no change in the b-wave:a-wave amplitude ratio (Fig. 2) indicating that the increase or decrease in the ERG was not selective and that the lesion causing this change occurred at the level of the photoreceptor. Secondly, the oscillatory potentials (Fig. 3 ) changed

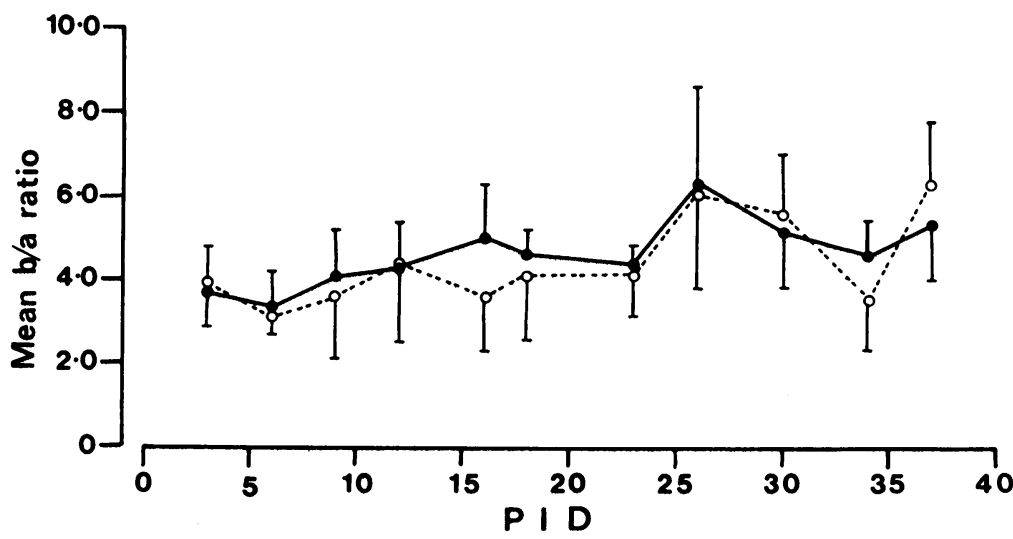


A
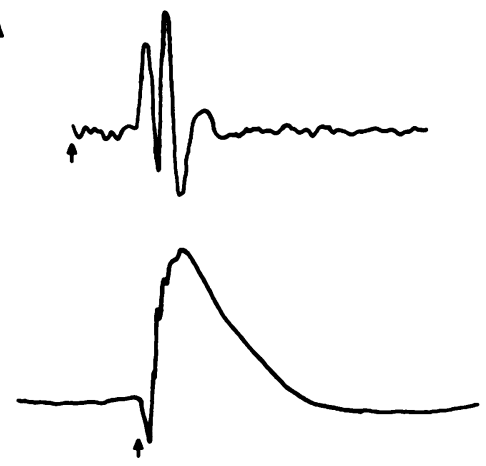

C

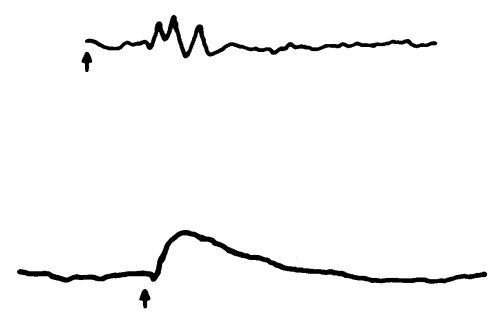

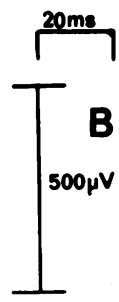
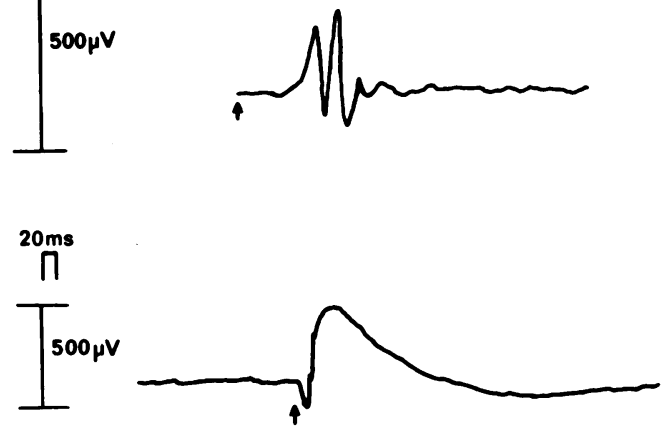

D

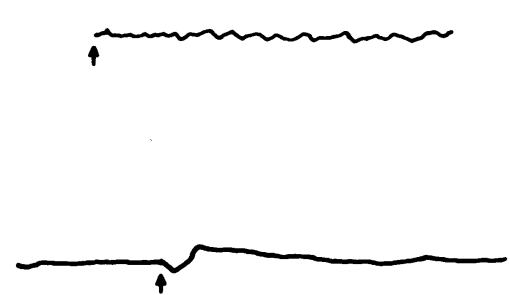

Fig. 3 Oscillatory potentials (upper trace of each set) and corresponding ERGs (lower traces). The oscillatory potentials were recorded via a high-pass filter. From control (B) and experimental animal E3 at PID 18 (A), E1 at PID $34(C)$ and E2 at PID $18(D)$. Arrows indicate stimulus. Note calibration changes.

according to the state of the whole ERG. Thus when the ERG became supernormal the oscillatory potentials increased in size, and when it was subnormal they were abolished. Thirdly, there was no change in either the a-wave or b-wave implicit times (measured from the stimulus onset to the peak of the wave) throughout the experiment (Fig. 4). Finally, the ERG flicker fusion frequency (EFFF) showed a gradual decline over the study period in experimental animals (Fig. 5). EFFF is a measure of the temporal resolution of the retina and is measured by the stimulating frequency at which the ERG wavelets became indistinguishable from the baseline.

Figs. 6A, B, C, D illustrate the histological features found in a control animal and an experimental animal (E2) at the end of the study period. The animal with active disease (E1) showed 1+ vitreous cells and an active retinal perivasculitis with some focal photo-

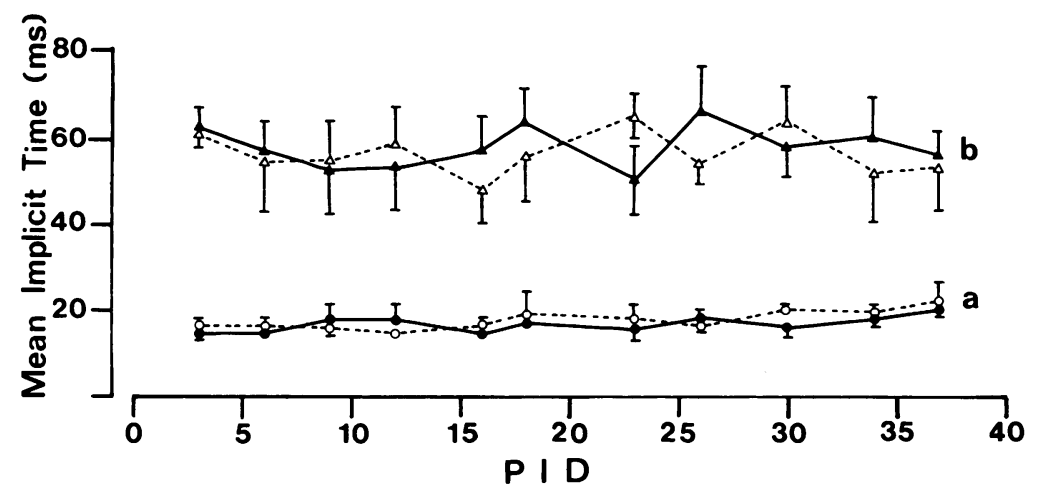

Fig. 4 Implicit times (mean $\pm S D$ ) of the a-wave (circles) and b-wave (triangles) for control (solid line) and experimental animals (dashed line) at $O$ Lu at days after inoculation. 
Fig. 5 ERG flicker fusion frequency (mean $\pm S D$ ) for control (solid line) and experimental (dashed line) animals at $0 \mathrm{Lu}$ (triangles), 2 Lu (squares), and 4 Lu (circles) at days after inoculation.

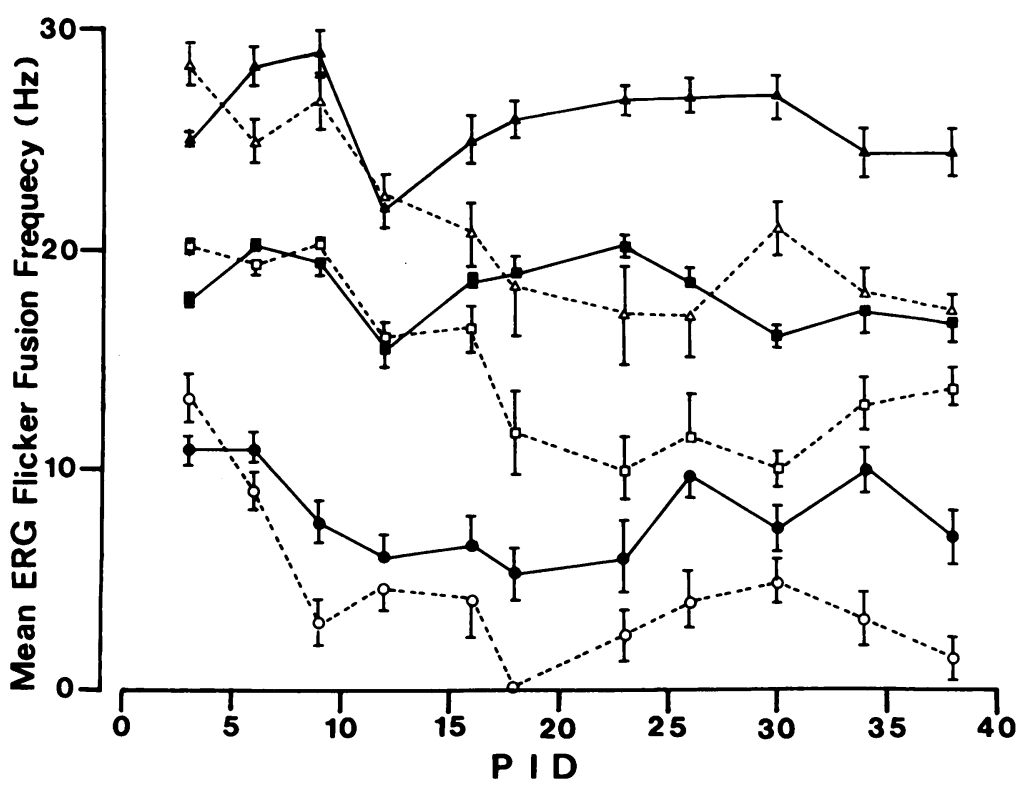

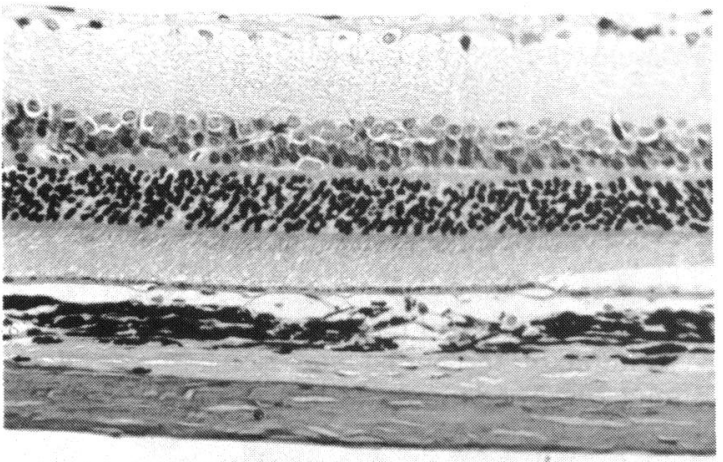

Fig. 6A

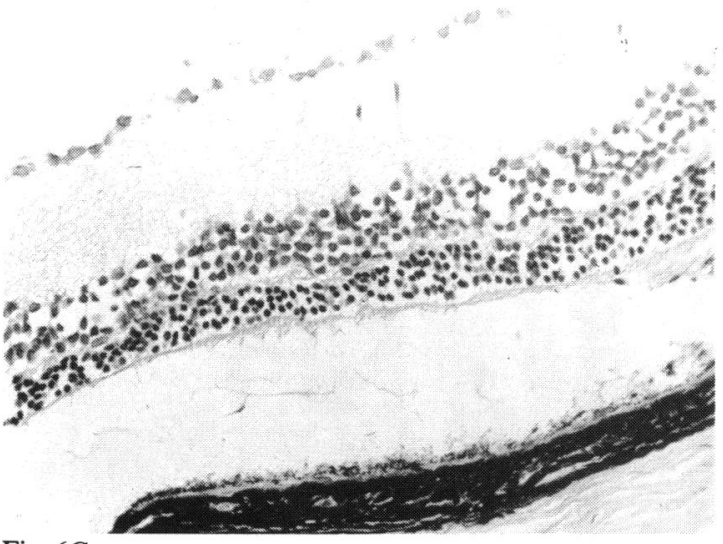

Fig. 6C

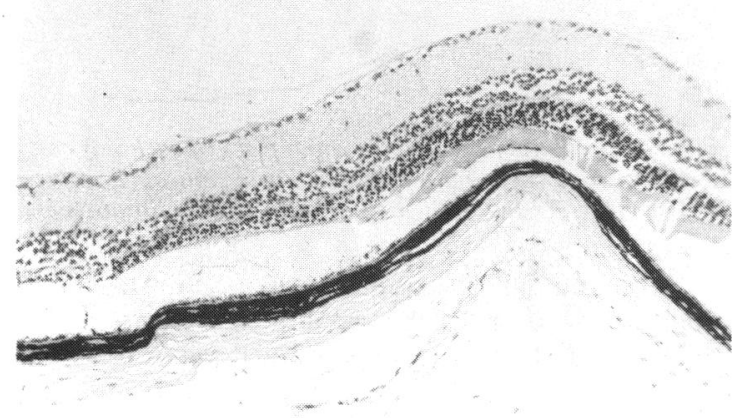

Fig. 6B

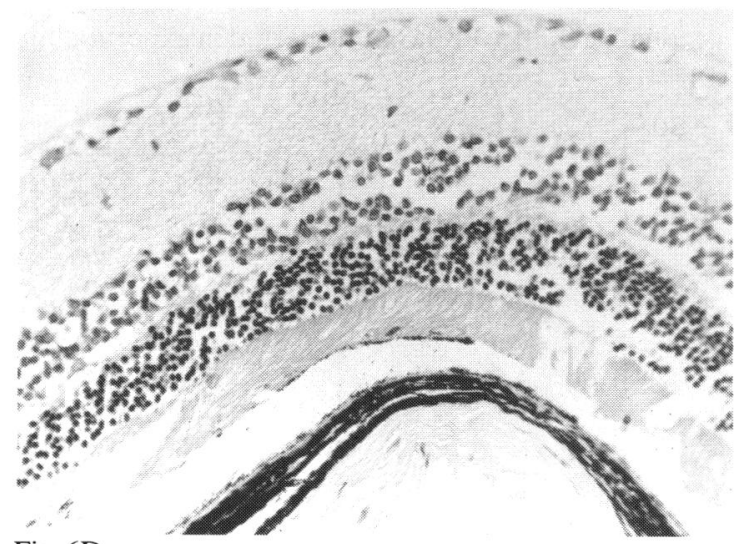

Fig. 6D 
receptor loss, while the third animal (E3) showed no abnormality.

GROUP II: THE SUPERNORMAL RESPONSE CLINICOPATHOLOGICAL CORRELATIONS

This experiment was designed to see if all animals produced a supernormal ERG prior to the onset of disease and whether this correlated with any underlying pathological features. ERGs were recorded on a daily basis from day 13 after inoculation, and each animal was killed when the ERG became supernormal. A supernormal ERG was defined as that showing larger b-wave amplitudes than controls at all intensity levels with $95 \%$ confidence limits. It was found that all animals' ERGs went through this stage, with a peak incidence on day 15 (Fig. 7). Again, the appearance of a supernormal ERG was associated with enhanced oscillatory potentials but a reduction in EFFF.

Histological examination of the eyes in which the ERG became supernormal was performed so that the examiner did not know the results of the ERGs. No abnormalities of the retina were found, and, although significant numbers of vitreous cells were found in some eyes, no correlation could be made between this number and the degree of supernormality of the ERG.

\section{GROUP III: THE SUBNORMAL RESPONSE AND RECOVERY}

Two groups of animals were used to assess the subnormal ERG and its subsequent recovery. In the first group of five animals three had suffered bilateral posterior uveitis by day 28 after inoculation and their ERGs were extinct at 120 days. In the other two animals, where uveitis was not observed within 28 days, the ERG was subnormal at 120 days. Histological examination of the eyes of these animals showed complete photoreceptor loss with glial replacement (Fig. 8) where the ERG was extinct, while in those where the ERG was subnormal there was focal photoreceptor destruction. The other layers of the retina, the pigment epithelium and choroid, appeared normal. There was no evidence of continuing inflammation in any eye as judged by the presence of inflammatory cells.

Fig. 6 A: Photomicrograph of peripheral retina taken from a control animal with a normal ERG (Haematoxylin and eosin, $\times 200$ ). B: Photomicrograph of peripheral retina taken from an animal with a subnormal ERG after disease had settled. Large areas of photoreceptor outer segment are destroyed (H and E $\times 100)$. C: Enlargements of the affected areas show concomitant thinning of the outer nuclear and plexiform layers, whereas D: there are areas of relatively normal retina ( $H$ and $E$ ). The retina is artefactually detached. Pigment epithelium and choroid appear normal $(\times 200)$.

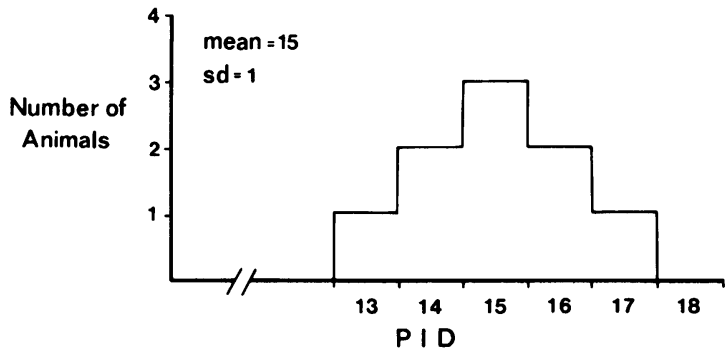

Fig. 7 Histogram showing the number of animals that went supernormal in at least one eye for each PID.

The subnormal response was analysed further in a group of 15 animals (10 experimental, five controls) in a longitudinal study until day 86 after inoculation. Five animals with subnormal ERGs on day 21 were killed and the remaining animals had their ERGs recorded every 20 days. Fig. 9 shows the change in the ERG b-wave amplitudes at five different light intensities for the animals that were followed up. In all experimental animals there was a significant fall in the ERG on day 21 with variable recovery after this. Thus some animals returned to control values, while others remained persistently subnormal. The subnormal ERG was also compared against control values as a function of relative log intensity of flash. Fig. 10 shows that the amplitude of the experimental animals' ERGs fell below that of the control animals at all intensities, and that the greater the intensity the greater the fall, as shown by a lower slope in ERG increase.

\section{Discussion}

We have demonstrated that there is a close correlation between the changes in ERG function and the

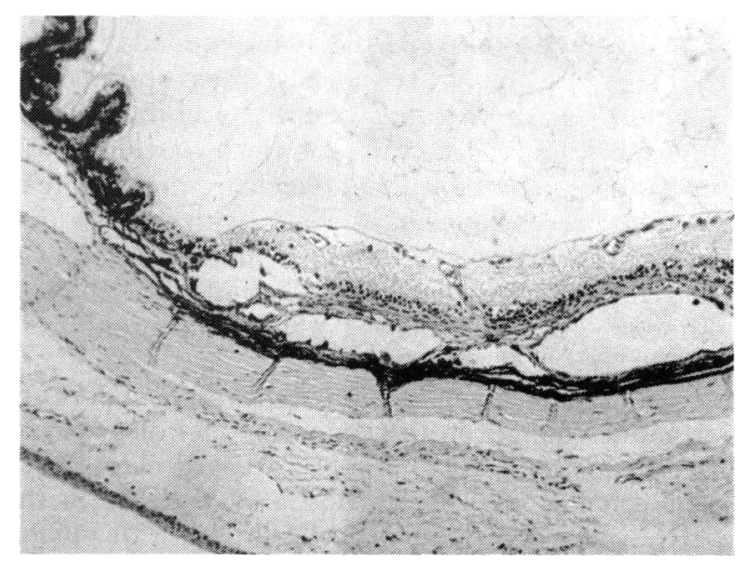

Fig. 8 Photomicrograph to show end stage disease in a severely affected rat which had an extinct ERG. The photoreceptors are completely replaced by fibrous tissue and there is some thinning of the inner nuclear layer. $H$ and $E$, $\times 200$. 
Fig. 9 b-Wave amplitude (mean士SE) for controls at PID 21 (circles) and experimental animals (squares) at PIDS 21, 37, 59, and 86 at five intensities $(\mathrm{Lu})$. Student's $t$ test compared the values of control and experimental animals. ${ }^{* * * *} p<0.0005,{ }^{* * *} p<0.005$, ${ }^{* *} p<0.025,{ }^{*} p<0.05$.
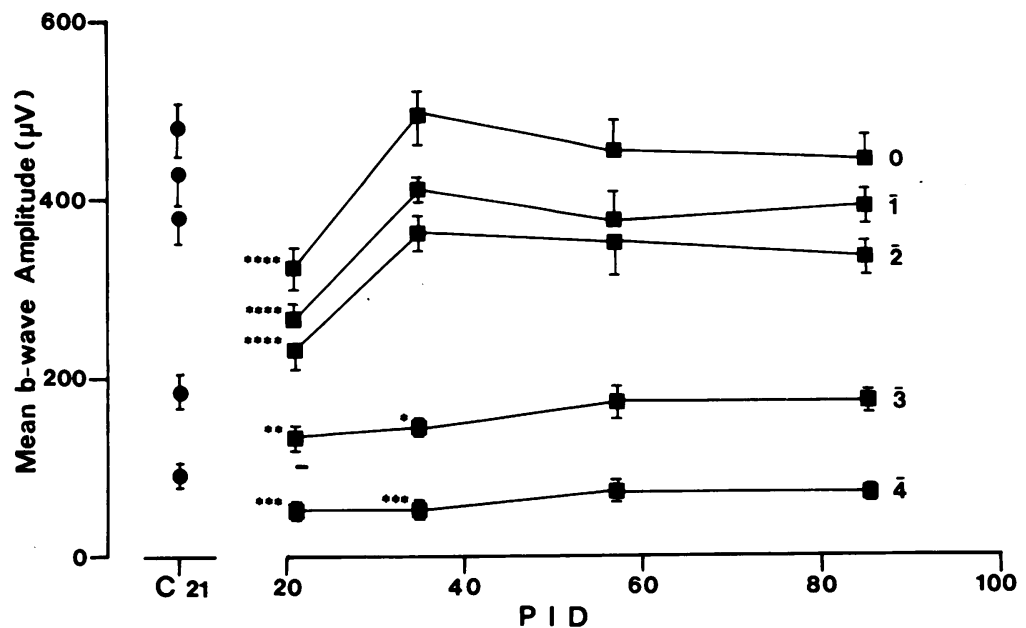

pathological changes of posterior uveitis in black hooded Lister rats produced by inoculation of retinal S-antigen. The ERGs underwent a supernormal stage 13-18 days after inoculation, when no pathology of the retina was evident. The ERGs then became rapidly subnormal as pathological changes developed in the photoreceptors, and recovered slowly to approach control levels with clinical recovery of the disease. The degree of subnormality of the ERG correlated with the extent of photoreceptor destruction when disease had settled. Even when clinical recovery from the disease had occurred, focal photoreceptor destruction was still apparent in histological sections of eyes from those animals which showed persistently subnormal ERGs.

The ocular inflammation induced by the inoculation of retinal S-antigen in the black hooded Lister rat primarily affected the retina. The disease produced a marked retinal vasculitis confirmed both clinically and angiographically. The retinal vasculitis was accompanied by a focal mononuclear cell infiltrate in the photoreceptor layer associated with necrosis and, in very severe disease, the whole photoreceptor layer was replaced by glial tissue. Pathological changes affecting the iris, ciliary body, and choroid were rarely seen. ${ }^{17}$

The physiological function of the retina underwent dynamic changes in this animal model of posterior uveitis as the ERGs initially increased before they significantly decreased. The initial increase in the ERG often coincided with the presence of inflammatory cells in the vitreous, but no clinical or. histological signs of, retinal disease were seen. The increase in ERG amplitudes was associated with a reduction in the ERG flicker fusion frequency, which indicated that 'supernormality' of the ERG was a transiently occurring abnormal response of the retina.

Previous authors ${ }^{1214}$ have also noted supernormality of the ERG in experimental uveitis and in uveitis in man, ${ }^{9}$ the changes being most marked in the b-wave, indicating that the initial lesion may lie in the post-receptoral retina from where the b-wave is generated. Others, ${ }^{20}$ however, have reported an increase in the ERG c-wave associated with changes on electron microscopy in the retinal pigment epithelium (RPE), suggesting that the earliest site of disease is the apical border of the pigment epithelium, where the c-wave is generated..$^{21} \mathrm{We}$ found the amplitudes of both the $a$ - and b-waves were increased without any change in the b:a ratio. This would suggest that the initial lesion was likely to be at the level of the photoreceptor, from which retinal Santigen is derived.

The question arises why the ERG was supernormal before retinal disease became apparent. Since supernormality occurred in the third week after inoculation, when antibody titres reached their highest levels, ${ }^{17}$ we suggest that the supernormal ERG is due to some unidentified biochemical change associated with the activity of antibodies against retinal Santigen. There is now increasing evidence that retinal $\mathrm{S}$-antigen is identical to the $48 \mathrm{~K}$ protein involved in visual transduction. ${ }^{22}$ This protein is thought to cap off phosphorylated rhodopsin to maintain it in an inactive state. Antibodies against this protein therefore may mop up the available S-antigen and in turn allow an uninhibited reaction by activated rhodopsin. However, until these antibodies have been shown to react with S-antigen in vivo, this hypothesis remains speculative.

Our study also showed that the ERGs became 


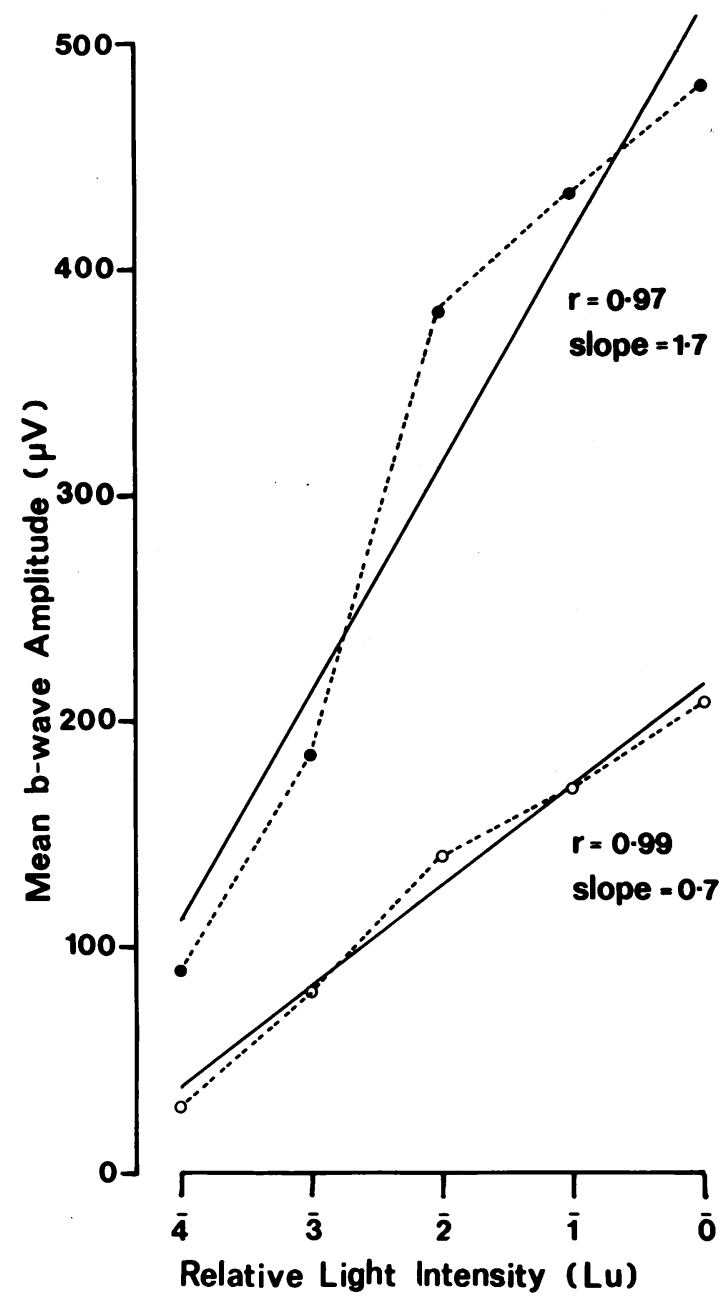

Fig. 10 Mean b-wave amplitude for control (closed circles) and experimental animals (open circles) on PID 21. Solid lines are best fits by regression analysis at the different light intensities. $r=$ correlation coefficient.

subnormal during active disease, but in most cases they recovered to a varying extent when the disease had settled. The depression of the ERG has been found by other authors studying EAU, ${ }^{11} 12^{14}$ is but they noted that the change was mostly confined to the b-wave, implicating the inner retinal layers. Furthermore similar changes have been found in man. ${ }^{569}$ This pattern of ERG change would also have been expected in this study, since the earliest sign of disease observed histologically was a perivasculitis of the retinal vessels which supply the retinal structures that generate the b-wave. However, we found that both the initial increase and subsequent depression of the ERG occurred without affecting the $b$ :a ratio. The decrease in both a- and b-wave amplitudes of the
ERG predicts that the lesion responsible affects the photoreceptor layer, and this was confirmed histologically as the main pathology in this animal model was destruction of the photoreceptors. Furthermore, two functions of the ERG predicted that this destruction was a focal rather than a diffuse process. Firstly, the implicit times of the a- and b-waves did not change, and this has been shown to correlate with focal rather than diffuse patterns of retinal disease. ${ }^{2324}$ Secondly, the slope of the intensity/ amplitude function was reduced, suggesting a loss of functional retinal area rather than overall retinal sensitivity loss. Moreover in cases when the ERGs were extinguished a complete photoreceptor loss was apparent.

We noted that in the majority of animals the ERGs recovered after the disease had settled. The degree of this recovery ranged from none at all, when the photoreceptor layer was completely destroyed, to control values, when the photoreceptor layer was largely unaffected. This finding implies that other mechanisms induced by inflammation in the retina must contribute reversibly to the depression of the ERG during active disease. It is also important to note that the good correlation between the degree of ERG loss and the extent of photoreceptor destruction was found when the disease was inactive. On the basis of these observations we suggest that the measurement of ERG functions when the disease is inactive may play an important role in estimating the extent of photoreceptor damage in posterior uveitis.

The authors thank Professor H Ikeda for the use of her laboratories, useful discussions, and critical review of the manuscript, $\mathrm{Mr} \mathrm{R}$ Dewhirst for help in preparing the illustrations, and Mrs G Blake for secretarial assistance. Retinal S-antigen was kindly provided by $\mathrm{Dr}$ E Kasp, Department of Immunology, St Thomas's Hospital. This work was supported in part by grants from the Medical Research Council (grant no. G 8303319 SB), the TFC Frost Charitable Trust, and the Research (Endowments) Committee, St Thomas's Hospital. JR is supported by a grant from the Special Trustees of St Thomas's Hospital to Professor H Ikeda. MRS holds a Training Awards Fellowship from the Medical Research Council.

\section{References}

1 Brown KT. The Electroretinogram: its components and their origins. Vision Res 1968; 8: 633-77.

2 Tomita T, Yanagida T. Origins of the ERG waves. Vision Res 1981; $21: 1703-7$.

3 Ikeda H. Clinical electroretinography. In: Halliday AM, ed. Evoked potentials and clinical testing. London: Churchill Livingstone, 1982: 121-47.

4 Algvere P. Electroretinographic studies on posterior uveitis. Acta Ophthalmol (Kbh) 1967; 45: 299-313.

5 Hatt M, Niemeyer G. Elektroretinographie bei morbus Behçet. Graefes Arch Klin Exp Ophthalmol 1976; 198: 113-20.

6 Karpe G. The electroretinogram under pathological conditions. Acta Ophthalmol (Kbh) 1945; 24 (suppl): 69-90.

7 Straub W. Das electroretinogramm. Stuttgart: Enke, 1961.

8 Yonemura D, Tsuzuki K, Aoki T. Clinical importance of the oscillatory potential in the human ERG. Acta Ophthalmol (Kbh) 1962; 70 (suppl): 115-23. 
9 François J. L'electrorétinographie dans les uvéites. Ophthalmologica 1953; 125: 137-43.

10 Takata H. Electroretinographic studies on experimental uveitis. Report 1: ERG in experimental uveitis. Nippon Ganka Gakkai Zasshi 1963; 67: 1814-27.

11 Algvere P, Hedin A, Kock E. The electroretinogram and histopathology in experimental uveitis of the pigeon. Acta Ophthalmol (Kbh) 1968; 46: 920-35.

12 Takata H. Electroretinographic studies of experimental uveitis. Report II. Analysis of 'Inflammation type ERG'. Nippon Ganka Gakkai Zasshi 1963; 67: 1829-37.

13 Lawwill T, Wacker W, Macdonald R. The role of electroretinography in evaluating posterior uveitis. Am J Ophthalmol 1972; 74: 1086-93.

14 Macdonald R. Correlative studies on experimental allergic uveoretinal disease. Trans Am Ophthalmol Soc 1971; 69: $397-439$.

15 Wong VG, Green WR. Rhodopsin and blindness. Trans Am Ophthalmol Soc 1977; 75: 272-82.

16 Wacker WB, Donoso LA, Kalsow CM, Yankeelov JA, Organisciak DT. Experimental allergic uveitis: isolation, characterisation and localisation of a soluble uveito-pathogenic antigen from bovine retina. J Immunol 1977; 119: 1949-58.

17 Stanford MR, Brown EC, Kasp E, Graham EM, Sanders MD, Dumonde DC. Experimental posterior uveitis. I: Clinical, angiographic, and histopathological features. Br J Ophthalmol 1987; 71: $585-92$.
18 Kasp E, Banga JP, Sanders MD, Dumonde DC. A novel approach for the purification of human, porcine and bovine S-antigen utilising hydrophobic absorption chromatography. Invest Ophthalmol Vis Sci 1985; 26 (suppl): 97. ARVO abstracts.

19 Sasovetz D. Ketamine hydrochloride. An effective general anesthetic for use in electroretinography. Ann Ophthalmol 1978; 10: $1510-4$.

20 Asayama R, Yoshida E, Yano T, Ikeda K, Nagata M. Experimental studies on the significance of some components of the clinical ERG. In: François J, ed. The clinical value of electroretinography. ISCERG Symp. Ghent 1966. Basel: Karger, 1968: 171-83.

21 Brown KT, Wiesel TN. Localisation of origins of electroretinogram components by intraretinal recording of the intact cat eye. J Physiol (Lond) 1961; 158: 257-80.

22 Pfister C, Chabre M, Tuyen VV, et al. Retinal S-antigen identified as the $48 \mathrm{~K}$ protein regulating light-dependent phosphodiesterase in rods. Science 1985; 228: 891-3.

23 Berson EL, Gouras MD, Hoff M. Temporal aspects of the electroretinogram. Arch Ophthalmol 1969; 81: 207-14.

24 François J, de Rouck A. Behaviour of the ERG and EOG in localised retinal destruction by photocoagulation. In: Burian HM, Jacobson JH, eds. Clinical electroretinography. London: Pergamon, 1966: 191-202.

Accepted for publication 4 December 1986. 